\title{
CUP PRODUCTS AND GROUP EXTENSIONS
}

\author{
P. A. LINNELL \\ (Received 30 June 1989; revised 19 December 1989) \\ Communicated by J. H. Rubinstein
}

\begin{abstract}
Let $G$ be a finitely generated group and let $R$ be a commutative ring, regarded as a $G$-module with $G$ acting trivially. We shall determine when the cup product of two elements of $H^{1}(G, R)$ is zero. Our method will use the interpretation of $H^{2}(G, R)$ as extensions of $G$ by $R$. This will give an alternative demonstration of results of Hillman and Würfel.
\end{abstract}

1980 Mathematics subject classification (Amer. Math. Soc.) (1985 Revision): primary $20 \mathrm{~J} 05$; secondary 20 E 22.

Keywords and phrases: cup product, group extension.

\section{Introduction}

Throughout this paper $G$ is a finitely generated group, $p$ is 0 or a prime, and $k=\mathbb{Z} / p \mathbb{Z}$ regarded as a $\mathbb{Z} G$-module with $G$ acting trivially. The kernel of the cup product $\cup: H^{1}(G, k) \otimes H^{1}(G, k) \rightarrow H^{2}(G, k)$ was studied in [3], [4] and [7], these papers depending in part on cochain calculations. We shall offer a different approach, using the interpretation of $H^{2}(G, k)$ as extensions of $G$ by $k$. With the exception of Theorem 2(ii), our results are essentially those of [4] and [7].

For any group $H$ we set $H^{*}=H^{\prime} H^{p}$ when $p \neq 0$. As usual, we shall identify $H^{1}(H, k)$ with $\operatorname{Hom}(H, k)$. If $H$ is nilpotent, then $\tau(H)$ will indicate the torsion subgroup of $H$ (in other words the elements of finite order in $H$ ), and when $p=0$, we define $G^{*}$ by $G^{*} / G^{\prime}=\tau\left(G / G^{\prime}\right)$.

(C) 1991 Australian Mathematical Society $0263-6115 / 91 \$ A 2.00+0.00$ 
Let $H^{1}(G, k) \wedge H^{1}(G, k)$ denote the alternating product, and $H^{1}(G, k) \odot$ $H^{1}(G, k)$ the symmetric product. Since the cup product is anticommutative, it induces homomorphisms

$$
\gamma: H^{1}(G, k) \wedge H^{1}(G, k) \rightarrow H^{2}(G, k) \quad \text { if } p \neq 2,
$$

and

$$
\theta: H^{1}(G, k) \odot H^{1}(G, k) \rightarrow H^{2}(G, k) \quad \text { if } p=2,
$$

as described in [3]. We shall prove

Theorem 1. Suppose $p \neq 0$, and $f, g \in \operatorname{Hom}(G, k)$ are linearly independent over $k$ with kernels $H, K$ respectively.

(i) $f \cup g=0$ if and only if $H^{*} K^{*} \neq G^{*}$.

(ii) If $p \neq 2$, then $\operatorname{ker} \gamma \cong G^{*} /\left[G^{*}, G\right] G^{p}$.

(iii) If $p=2$, then $\operatorname{ker} \theta \cong G^{*} /\left[G^{*}, G\right] G^{* *}$.

THEOREM 2. Let $p=0$, let $f, g \in \operatorname{Hom}(G, \mathbb{Z})$ be linearly independent over $\mathbb{Z}$, and let $H=\operatorname{ker} f \cap \operatorname{ker} g$.

(i) If $K /\left[G^{*}, G\right]=\tau\left(G /\left[G^{*}, G\right]\right)$, then $\operatorname{ker} \gamma \cong G^{*} / K$.

(ii) Suppose $r$ is the index of $\langle\bar{f}, \bar{g}\rangle$ in $H^{1}(G / H, \mathbb{Z})$, where $\bar{f}$ and $\bar{g}: G / H \rightarrow \mathbb{Z}$ are the homomorphisms induced by $f$ and $g$ respectively. If $T /[H, G]=\tau(G /[H, G])$, then $f \cup g$ has finite additive order in $H^{2}(G, \mathbb{Z})$ if and only if $G^{\prime} /[H, G]$ is infinite, and in this case the order is $\frac{1}{r}$ l. c. m. $\left(r,\left|G^{*} / G^{\prime} T\right|\right)$.

We use the following method: as in [4] we consider the five term exact sequence associated with the group extension $1 \rightarrow G^{*} \rightarrow G \rightarrow G / G^{*} \rightarrow 1$ :

$0 \rightarrow H^{1}\left(G / G^{*}, k\right) \rightarrow H^{1}(G, k) \rightarrow H^{1}\left(G^{*}, k\right)^{G} \stackrel{\delta}{\rightarrow} H^{2}\left(G / G^{*}, k\right) \rightarrow H^{2}(G, k)$.

It will be important to describe the map $\delta$ accurately. This will be done by using group extensions (Lemma 3 ) and the well known structure of $H^{2}\left(G / G^{*}, k\right)$ (Lemmas 4 and 5 ). The motivation for this paper was to show that the approach of [4] could be modified so as to avoid complicated cochain calculations.

\section{Notation}

Mappings will mostly be written on the left, and modules will be left modules. Let $A, B \leq H$ be groups, let $X \subseteq H$, and let $M$ be a $\mathbb{Z} H$ module. Then we use the notation $H^{\prime}$ for the commutator subgroup of 
$H,\langle X\rangle$ for the subgroup generated by $X,|X|$ for the order $X,[A, B]$ for $\left\langle a^{-1} b^{-1} a b \mid a \in A, b \in B\right\rangle$, and $M^{H}$ for $\{m \in M \mid h m=m$ for all $h \in H\}$. The restriction map from $H^{2}(H, M)$ to $H^{2}(A, M)$ will be denoted by res $_{H, A}$, and the lowest common multiple of two positive integers by l.c. $\mathrm{m}$. If $\theta$ is a map, then $\operatorname{im} \theta$ will indicate the image of $\theta$, and $\operatorname{ker} \theta$ the kernel of $\theta$. Suppose $A, B \triangleleft H, A$ is abelian and $B$ acts trivially on $M$. Then we can also view $M$ as a $\mathbb{Z}[H / B]$-module, and we can make $A$ into a $\mathbb{Z} H$-module by letting $H$ act via conjugation so that $h \cdot a=h a h^{-1}$ for $a \in A$ and $h \in H$; we shall use these well known observations without further comment in the future.

\section{Preliminary results}

Most of the lemmas in this section are well known. For the purposes of this paper, the theory on page 294 of [2] instead of Lemma 3 would be sufficient.

LEMMA 3. Let $A$ be an abelian normal subgroup of the group $H$, let $K=$ $H / A$, let $M$ be a $\mathbb{Z} K$-module, and view $A$ as a $\mathbb{Z} K$-module with $K$ acting on $A$ by conjugation. Let $f \in \operatorname{Hom}_{\mathrm{zK}}(A, M)$ and let

$$
\delta: H^{1}(A, M)^{H}=\operatorname{Hom}_{\mathrm{z} K}(A, M) \rightarrow H^{2}(K, M)
$$

be the transgression map associated with the group extension $1 \rightarrow A \rightarrow H \rightarrow$ $K \rightarrow 1$. Suppose $\chi: K \times K \rightarrow A$ is a factor set representing the element in $H^{2}(K, A)$ corresponding to the above extension. Then (after choosing the notation correctly) $-\delta(f)$ is an element of $H^{2}(K, M)$ which is represented by the factor set $f \chi: K \times K \rightarrow M$. In particular if $f$ is surjective, then $\delta(f)$ is represented by a group extension of the form

$$
1 \rightarrow A / \operatorname{ker} f \rightarrow H / \operatorname{ker} f \rightarrow K \rightarrow 1 \text {. }
$$

Proof. To ensure that $-\delta(f)$ and $f \chi$ represent the same element in $H^{2}(K, A)$, we need to choose the notation correctly, and the notation of [6, IV.4 and XI.9] will suffice. Let $T$ be a set of coset representatives for $A$ in $H$, let ${ }^{-}: H \rightarrow K$ denote the natural epimorphism, and let $B(\mathbb{Z} H)$ denote the (normalized) bar resolution [6, page 114]. Thus $B_{n}(\mathbb{Z} H)$ is the free $\mathbb{Z} H$ module with free generators $\left\{\left[x_{1}|\ldots| x_{n}\right] \mid x_{i} \in H \backslash 1\right\}$, and $f$ is represented by any $\hat{f} \in \operatorname{Hom}_{\mathrm{z} H}\left(B_{1}(\mathbb{Z} H), M\right)$ such that $\hat{f}([a])=f(a)$ for all $a \in A \backslash 1$; we shall define $\hat{f}$ by $\hat{f}([a t])=f(a)$ for all $\alpha \in A$ and $t \in T \quad(a t \neq 1)$, and assume $1 \in T$. Let $\partial: B_{2}(\mathbb{Z} H) \rightarrow B_{1}(\mathbb{Z} H)$ be the boundary map defined by

$$
\partial([x \mid y])=x[y]-[x y]+[x]
$$


for $x, y \in H \backslash 1$. Then $\delta(f)$ is represented by the factor set $\psi: K \times K \rightarrow M$ satisfying $\psi(\bar{x}, \bar{y})=\hat{f} \partial([x \mid y])$ for $x, y \in H$ (cf. the "connection" of [6, page 349]). In fact if we write $x=a r, y=b s, x y=c t \quad(a, b, c \in$ $A ; r, s, t \in T)$, then $\psi(\bar{x}, \bar{y})=f\left(t s^{-1} r^{-1}\right)$. But the factor set $\chi$ can be defined by $\chi(\bar{x}, \bar{y})=r s t^{-1}$ (see [6, page 111]), and we deduce that $-\delta(f)$ and $f \chi$ represent the same element of $H^{2}(K, M)$.

Now suppose $f$ is surjective. If $f \chi$ is represented by an extension of the form $1 \rightarrow M \rightarrow E \rightarrow K \rightarrow 1$, then there exists a commutative diagram

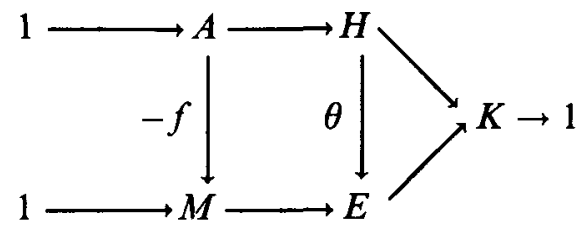

for some group homomorphism $\theta: H \rightarrow E$, necessarily surjective, and the result follows.

LeMMA 4. Let $p$ be a prime, let $G$ be a finite elementary abelian p-group, and let $\left(f_{1}, \ldots, f_{n}\right)$ be a $k$-basis for $H^{1}(G, k)$.

(i) If $p=2$, then the set $\left\{f_{i} \cup f_{j} \mid 1 \leq i, j \leq n\right\}$ is a $k$-basis for $H^{2}(G, k)$.

(ii) If $p$ is odd, then the set $\left\{f_{i} \cup f_{j}, \beta f_{l} \mid 1 \leq i<j \leq n, 1 \leq l \leq n\right\}$ is a $k$ basis for $H^{2}(G, k)$, where $\beta: H^{1}(G, k) \rightarrow H^{2}(G, k)$ is the Bockstein map. In particular if $\chi \in H^{2}(G, k)$, then $\operatorname{res}_{G, A} \chi=0$ for all cyclic subgroups $A$ of $G$ if and only if $\chi=\sum_{i<j} \lambda_{i j} f_{i} \cup f_{j}$ for some $\lambda_{i j} \in k$.

LeMma 5. Let $G$ be a free abelian group, and let $\left(f_{1}, \ldots, f_{n}\right)$ be a $\mathbb{Z}$ basis for $H^{1}(G, \mathbb{Z})$. Then the set $\left\{f_{i} \cup f_{j} \mid 1 \leq i<j \leq n\right\}$ is a $\mathbb{Z}$-basis for $H^{2}(G, \mathbb{Z})$.

Proof. Lemmas 4 and 5 follows from the Künneth theorem (see [1, page $101]$ and [5, VI.15]). For information of the Bockstein map, see [1, 2.23].

\section{Cup products and group extensions}

Lemma 6. Let $p$ be a prime, let $G$ be an elementary abelian p-group, and let $\left(f_{1}, f_{2}, \ldots, f_{n}\right)$ be a $k$-basis for $H^{1}(G, k)$. Write $K_{1}=\operatorname{ker} f_{1}$ and $K_{2}=\operatorname{ker} f_{2}$. Suppose $\chi \in H^{2}(G, k)$ is represented by the group extension

$$
0 \rightarrow k \rightarrow E \stackrel{\theta}{\rightarrow} G \rightarrow 1 \text {. }
$$


(i) If $\theta^{-1}\left(K_{1}\right)$ and $\theta^{-1}\left(K_{2}\right)$ are elementary abelian, then $\chi=\lambda f_{1} \cup f_{2}$ for some $\lambda \in k$.

(ii) If $p$ is odd, then $E$ has exponent $p$ if and only if $\chi=\sum_{i<j} \lambda_{i j} f_{i} \cup f_{j}$ for some $\lambda_{i j} \in k$.

Proof. Suppose $\theta^{-1}\left(K_{1}\right)$ and $\theta^{-1}\left(K_{2}\right)$ are elementary abelian. By Lemma 4 we may write

$$
\chi=\sum_{i \leq j} \lambda_{i j} f_{i} \cup f_{j} \quad \text { if } p=2,
$$

and

$$
\chi=\sum_{i<j} \lambda_{i j} f_{i} \cup f_{j}+\sum_{i} \lambda_{i} \beta f_{i} \text { if } p \text { is odd, }
$$

where $\lambda_{i j}, \lambda_{i} \in k$. Since $\theta^{-1}\left(K_{1}\right)$ is elementary abelian, $\operatorname{res}_{G, K_{1}} \chi=0$ and we see that $\lambda_{i}=\lambda_{i j}=0$ if $i \neq 1$. Also $\theta^{-1}\left(K_{2}\right)$ is elementary abelian, hence $\operatorname{res}_{G, K_{2}} \chi=0$ and we deduce that $\lambda_{1}=\lambda_{1 j}=0$ if $j \neq 2$. This proves (i).

It is easy to show (and is well known) that $E$ has exponent $p$ if and only if $\operatorname{res}_{G, A} \chi=0$ for all cyclic subgroups $A$ of $G$. Thus we obtain (ii) from Lemma 4(ii).

LEMMA 7. Let $G$ be a free abelian group, let $\left(f_{1}, \ldots, f_{n}\right)$ be a $\mathbb{Z}$-basis for $H^{1}(G, \mathbb{Z})$, and let $K=\operatorname{ker} f_{1} \cap \operatorname{ker} f_{2}$. Suppose $\chi \in H^{2}(G, \mathbb{Z})$ is represented by the group extension

$$
0 \rightarrow \mathbb{Z} \rightarrow E \stackrel{\theta}{\rightarrow} G \rightarrow 1 \text {. }
$$

(i) If $\left[E, \theta^{-1}(K)\right]=1$, then $\chi=r f_{1} \cup f_{2}$ where either $r=0$ or $r=$ $\pm\left|\operatorname{ker} \theta / E^{\prime}\right|$.

(ii) If $\chi=r f_{1} \cup f_{2}$ where $r \in \mathbb{Z}$, then $\left[E, \theta^{-1}(K)\right]=1$.

Proof. By Lemma 5, write $\chi=\sum_{i<j} \lambda_{i j} f_{i} \cup f_{j}$ where $\lambda_{i j} \in \mathbb{Z}$, and set $L=\operatorname{ker} \theta$ and $K_{i}=\operatorname{ker} f_{i}(1 \leq i \leq n)$.

(i) Since $\theta^{-1}\left(K_{1}\right)$ is abelian, $\operatorname{res}_{G, K_{1}} \chi=0$ and we see that $\lambda_{i j}=0$ if $i \neq 1$, and then $\theta^{-1}\left(K_{2}\right)$ abelian imples that $\operatorname{res}_{G, K_{2}} \chi=0$, and hence $\lambda_{1 j}=0$ if $j \neq 2$. Thus $\chi=r f_{1} \cup f_{2}$ for some $r \in \mathbb{Z}$. Suppose $r= \pm 1$. If $E^{\prime} \neq L$, then there exists a prime $p$ such that $E^{\prime} \subseteq L^{p}$. Let $\pi: \mathbb{Z} \rightarrow \mathbb{Z} / p \mathbb{Z}$ denote the natural surjection. Since $\left(\pi f_{1}, \ldots, \pi f_{n}\right)$ is a $\mathbb{Z} / p \mathbb{Z}$-basis for $H^{1}(G, \mathbb{Z} / p \mathbb{Z})$, it follows from Lemma 4 that $\pi f_{1} \cup \pi f_{2} \neq 0$, and hence

$$
0 \rightarrow \mathbb{Z} / p \mathbb{Z} \rightarrow E / L^{p} \rightarrow G \rightarrow 1
$$

is nonsplit. This contradicts $E^{\prime} \subseteq L^{p}$. Therefore $E^{\prime}=L$. 
In general if $0 \neq r \in \mathbb{Z}$, let $\mu_{r}: \mathbb{Z} \rightarrow \mathbb{Z}$ denote multiplication by $r$. Then we have a commutative diagram

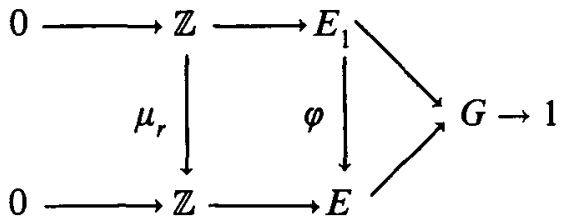

for some map $\varphi$, where the top sequence represents $f_{1} \cup f_{2}$ and the bottom sequence $r f_{1} \cup f_{2}$. This shows that $\left|L / E^{\prime}\right|=|r|$, and (i) follows.

(ii) Since res ${ }_{K_{1}} f_{1} \cup f_{2}=\operatorname{res}_{K_{2}} f_{1} \cup f_{2}=0$, we see that $\theta^{-1}\left(K_{1}\right)$ and $\theta^{-1}\left(K_{2}\right)$ are abelian, and hence $\left[\theta^{-1}\left(K_{1}\right) \theta^{-1}\left(K_{2}\right), \theta^{-1}(K)\right]=1$. But $[a, b]^{s}=$ $\left[a^{s}, b\right]$ for $a, b \in E, s \in \mathbb{Z}$, and $\theta^{-1}\left(K_{1}\right) \theta^{-1}\left(K_{2}\right)$ has finite index in $E$, and the proof of (ii) is easily completed.

Proof of Theorem 1. Consider the five term exact sequence associated with the group extension $1 \rightarrow G^{*} \rightarrow G \rightarrow G / G^{*} \rightarrow 1$ :

$0 \rightarrow H^{1}\left(G / G^{*}, k\right) \stackrel{\theta}{\rightarrow} H^{1}(G, k) \rightarrow H^{1}\left(G^{*}, k\right) \stackrel{\delta}{\rightarrow} H^{2}\left(G / G^{*}, k\right) \stackrel{\oplus}{\rightarrow} H^{2}(G, k)$

where $\theta$ and $\varphi$ are the inflation maps, and $\delta$ is the transgression map. Choose $\bar{f}, \bar{g} \in H^{1}\left(G / G^{*}, k\right)$ such that $\theta(\bar{f})=f, \theta(\bar{g})=g$. If $f \cup g=0$, then $\bar{f} \cup \bar{g}=\delta(u)$ for some $u \in H^{1}\left(G^{*}, k\right)^{G}$; note that $u \neq 0$, so $u$ is onto and $G^{*} / L \cong k$ where $L=\operatorname{ker} u$. Using Lemma 3 , we see that $\delta(u)$ is represented by a group extension of the form

$$
0 \rightarrow k \rightarrow G / L \stackrel{\pi}{\rightarrow} G / G^{*} \rightarrow 1
$$

for some homomorphism $\pi$. Since $\operatorname{res}_{G / G^{*}, H / G^{*}} \bar{f} \cup \bar{g}=0$, it follows that $\pi^{-1}\left(H / G^{*}\right)$ is elementary abelian, and hence $H^{*} \subseteq L$. Similarly $K^{*} \subseteq L$ and we deduce that $H^{*} K^{*} \neq G^{*}$.

Conversely suppose $H^{*} K^{*} \neq G^{*}$. Choose a subgroup $M$ such that $H^{*} K^{*}$ $\subseteq M<G^{*}$ and $M$ is maximal under these conditions. Then $M \triangleleft G$ and $G^{*} / M \cong k$ because $\left[G, G^{*}\right]=\left[H K, G^{*}\right]=\left[H, G^{*}\right]\left[K, G^{*}\right] \subseteq H^{*} K^{*}$. Since $H / M$ and $K / M$ are elementary abelian, application of Lemma $6(i)$ shows that

$$
0 \rightarrow k \rightarrow G / M \rightarrow G / G^{*} \rightarrow 1
$$

is represented by $\lambda \bar{f} \cup \bar{g}$ for some $\lambda \in k$. Now $G / M$ is not elementary abelian, hence $\lambda \neq 0$ and it follows from Lemma 3 that $\bar{f} \cup \bar{g} \in \operatorname{im} \delta$. Therefore $f \cup g=0$ which proves (i).

Now suppose $p$ is odd and let

$$
\bar{\gamma}: H^{1}\left(G / G^{*}, k\right) \wedge H^{1}\left(G / G^{*}, k\right) \rightarrow H^{2}\left(G / G^{*}, k\right)
$$


be the map induced by the cup product. Then $\bar{\gamma}$ is a monomorphism by Lemma 4(ii) and $\varphi \bar{\gamma}=\gamma(\theta \wedge \theta)$, and hence

$$
\operatorname{ker} \gamma \cong \operatorname{ker} \gamma(\theta \wedge \theta)=\operatorname{ker} \varphi \bar{\gamma} \cong \operatorname{ker} \varphi \cap \operatorname{im} \bar{\gamma}=\operatorname{im} \delta \cap \operatorname{im} \bar{\gamma}
$$

because $\theta$ is an isomorphism and $\operatorname{ker} \varphi=\operatorname{im} \delta$. Since $\delta$ is a monomorphism, we deduce that

$$
\operatorname{ker} \gamma \cong\left\{v \in H^{1}\left(G^{*}, k\right){ }^{G} \mid \delta(v)=\sum_{i<j} \lambda_{i j} f_{i} \cup f_{j} \text { for some } \lambda_{i j} \in k\right\} \text {. }
$$

If $v \neq 0$ and $N=\operatorname{ker} v$, then $\delta(v)$ is represented by an extension of the form

$$
0 \rightarrow k \rightarrow G / N \rightarrow G / G^{*} \rightarrow 1
$$

by Lemma 3. It now follows from Lemma 6(ii) that $G / N$ has exponent $p$ if and only if $\delta(v)=\sum_{i<j} \lambda_{i j} f_{i} \cup f_{j}$ for some $\lambda_{i j} \in k$. Therefore ker $\gamma \cong$ $\operatorname{Hom}\left(G^{*} /\left[G^{*}, G\right] G^{p}, k\right)$. But $G$ is finitely generated, hence $G^{*}$ is finitely generated and we conclude that $\operatorname{ker} \gamma \cong G^{*} /\left[G^{*}, G\right] G^{p}$ as required.

The case $p=2$ is similar but easier; one uses Lemma 4(i) instead of Lemmas 4(ii) and 6(ii). Since this argument is identical to that of $[4$, Section 3], we omit it.

Proof of Theorem 2. Consider the five term exact sequence associated with the group extension $1 \rightarrow G^{*} \rightarrow G \rightarrow G / G^{*} \rightarrow 1$ :

$$
\begin{aligned}
0 \rightarrow H^{1}\left(G / G^{*}, \mathbb{Z}\right) \stackrel{\theta}{\rightarrow} H^{1}(G, \mathbb{Z}) \rightarrow H^{1}\left(G^{*}, \mathbb{Z}\right)^{G} \\
\quad \stackrel{\delta}{\rightarrow} H^{2}\left(G / G^{*}, \mathbb{Z}\right) \stackrel{\varphi}{\rightarrow} H^{2}(G, \mathbb{Z}),
\end{aligned}
$$

where $\theta$ and $\varphi$ are the inflation maps, and $\delta$ is the transgression map. If $\bar{\gamma}: H^{1}\left(G / G^{*}, \mathbb{Z}\right) \wedge H^{1}\left(G / G^{*}, \mathbb{Z}\right) \rightarrow H^{2}\left(G / G^{*}, \mathbb{Z}\right)$ is the homomorphism induced by the cup product, then $\bar{\gamma}$ is an isomorphism by Lemma 5 , and $\varphi \bar{\gamma}=\gamma(\theta \wedge \theta)$. Since $\theta$ is an isomorphism, $\delta$ is a monomorphism and we deduce that

$$
\begin{aligned}
\operatorname{ker} \gamma & \cong \operatorname{ker} \gamma(\theta \wedge \theta)=\operatorname{ker} \varphi \bar{\gamma} \cong \operatorname{ker} \varphi=\operatorname{im} \delta \\
& \cong \operatorname{Hom}\left(G^{*} /\left[G^{*}, G\right], \mathbb{Z}\right) \cong G^{*} / K
\end{aligned}
$$

(because $G$ is finitely generated implies $G^{*} /\left[G^{*}, G\right]$ is finitely generated) which proves (i). The argument of this section is identical to that of $[4$, Section 2].

Now let $(e, h)$ be a $\mathbb{Z}$-basis for $H^{1}(G / H, \mathbb{Z})$. By anticommutativity of the cup product $e \cup e=h \cup h=0$, hence $f \cup g= \pm r e \cup h$, so we may assume that $r=1$ and that $(\bar{f}, \bar{g})$ is a $\mathbb{Z}$-basis for $H^{1}(G / H, \mathbb{Z})$. Choose a $\mathbb{Z}$-basis $\left(f_{1}, \ldots, f_{n}\right)$ of $H^{1}\left(G / G^{*}, \mathbb{Z}\right)$ such that $\theta\left(f_{1}\right)=f$ and $\theta\left(f_{2}\right)=g$. Suppose 
$f \cup g$ has finite additive order $s$. Then there exists $u \in \operatorname{Hom}\left(G^{*} /\left[G^{*}, G\right], \mathbb{Z}\right)$ such that $\delta(u)=s f_{1} \cup f_{2}$. Note that $u \neq 0$ so if $L=\operatorname{ker} u$, then $G^{*} / L \cong \mathbb{Z}$. Let $v: G^{*} \rightarrow \mathbb{Z}$ be an epimorphism with kernel $L$, so $u=t v$ for some $t \in \mathbb{Z}$. Then $t \delta(v)=s f_{1} \cup f_{2}$, thus $t \mid s$ by Lemma 5 and we deduce that $t= \pm 1$. Also application of Lemma 3 shows that $\delta(v)$ is represented by an extension of the form

$$
0 \rightarrow \mathbb{Z} \rightarrow G / L \rightarrow G / G^{*} \rightarrow 1 .
$$

Therefore $[H, G] \subseteq L$ and $\left|G^{*} / G^{\prime} L\right|=s$ by Lemma 7 . But it is easy to show that $G^{\prime} /[H, G]$ is cyclic, hence $L=T$ and we conclude that $\left|G^{*} / G^{\prime} T\right|=s$.

Conversely suppose $G^{\prime} /[H, G]$ is infinite. Since $G^{\prime} /[H, G]$ is cyclic, it follows that $G^{*} / T \cong \mathbb{Z}$. If $w: G^{*} \rightarrow \mathbb{Z}$ is an epimorphism with kernel $T$, then $\delta(w)$ is represented by an extension of the form

$$
0 \rightarrow \mathbb{Z} \rightarrow G / T \rightarrow G / G^{*} \rightarrow 1
$$

by Lemma 3. From Lemma 7(i), this extension also represents $\pm l f_{1} \cup f_{2}$ where $l=\left|G^{*} / G^{\prime} T\right|$. It follows that $l f \cup g=0$ and hence $f \cup g$ has finite order. This complete the proof of Theorem 2.

\section{References}

[1] D. J. Benson, Modular representation theory, (Lecture Notes in Mathematics, vol. 1081, Springer-Verlag, Berlin, New York, 1984).

[2] C. W. Curtis and I. Reiner, Methods of integral representation theory, vol. 1, (WileyInterscience, New York, 1981).

[3] J. A. Hillman, 'The kernel of the cup product', Bull. Austral. Math. Soc. 32 (1985), 261-274.

[4] J. A. Hillman, 'The kernel of integral cup product', J. Austral. Math. Soc. (Ser. A) 43 (1987), 10-15.

[5] P. J. Hilton and U. Stammbach, A course in homological algebra (GTM 4, SpringerVerlag, Berlin, New York, 1971).

[6] S. Mac Lane, Homology, (Springer-Verlag, Berlin, New York, 1975).

[7] T. Würfel, 'A note on the cup product for pro- $p$ groups', Proc. Amer. Math. Soc. 102 (1988), 809-813.

\section{Department of Mathematics}

Virginia Polytechnic Institute and State University

Blacksburg, Virginia 24061-0123.

U.S.A.

E-mail address: LINNELL@VTMATH.BITNET 